\title{
Hyperinsulinemia induces hypertension associated with neurogenic vascular dysfunction resulting from abnormal perivascular innervations in rat mesenteric resistance arteries
}

\author{
Yoshito Zamami ${ }^{1,2}$, Shingo Takatori ${ }^{1}$, Narumi Hobara ${ }^{3}$, Nana Yabumae ${ }^{1}$, Panot Tangsucharit ${ }^{1}$, Xin Jin $^{1}$, \\ Naoya Hashikawa ${ }^{3}$, Yoshihisa Kitamura ${ }^{4}$, Kenji Sasaki ${ }^{2}$ and Hiromu Kawasaki ${ }^{1}$
}

We previously reported that chronic hyperinsulinemia and insulin resistance induced by fructose-drinking loading elicited hypertension associated with abnormal neuronal regulation of vascular tone in an in vivo study using pithed rats. Therefore, to further clarify the detailed mechanisms of perivascular nervous system malfunction induced by chronic hyperinsulinemia, we investigated the neurogenic vascular responses and distribution of perivascular nerves using mesenteric vascular beds isolated from fructose-loaded rats with hyperinsulinemia. Male Wistar rats ( 6 weeks old) received $15 \%$ fructose solution as drinking fluid for 10 weeks (fructose-drinking rats, FDR), which resulted in significant increases in plasma levels of insulin, the glucose-insulin index, blood norepinephrine (NE) levels and systolic blood pressure, but not blood glucose levels, when compared with normal water-drinking rats (control rats). In perfused mesenteric vascular beds of FDR, enhanced adrenergic nerve-mediated vasoconstriction with no effect on NE-induced vasoconstriction and decreased calcitonin gene-related peptide (CGRP)-containing nerve-mediated vasodilation with no effect on CGRP-induced vasodilation were observed. Immunohistochemistry studies showed increased density of neuropeptide $Y$ immunopositive adrenergic fibers and reduced density of CGRP immunopositive fibers in mesenteric arteries of FDR. Furthermore, FDR showed decreased CGRP content in dorsal root ganglia. These findings suggest that dysfunction of the neuronal vascular control system resulting from abnormal innervation of mesenteric perivascular nerves induced by the hyperinsulinemic state is responsible for the development of hypertension in FDR.

Hypertension Research (2011) 34, 1190-1196; doi:10.1038/hr.2011.97; published online 28 July 2011

Keywords: adrenergic nerve; calcitonin gene-related peptide-containing nerve; chronic hyperinsulinemia; mesenteric resistance arteries

\section{INTRODUCTION}

Many clinical studies have shown the potential relationship between insulin resistance and hypertension, as patients with type 2 (noninsulin dependent) diabetes mellitus are frequently complicated by hypertension. ${ }^{1-3}$ Modan et al. ${ }^{4}$ suggested that there is a relationship between insulin levels and blood pressure, and several groups have hypothesized that insulin resistance and/or hyperinsulinemia contribute to the pathogenesis of hypertension. ${ }^{5,6}$ Thus, insulin has an important role in pathophysiological regulation of the cardiovascular system.

Previously, in an in vivo study using pithed rats without vasoreflex or autonomic driving, we demonstrated that chronic loading with a high concentration of fructose solution in drinking water (fructose-drinking rats, FDR) resulted in an augmented adrenergic nerve-mediated vasoconstriction and a decreased calcitonin generelated peptide-containing (CGRPergic) nerve-mediated vasodilatation, which showed hyperinsulinemia with euglycemia and hypertension. ${ }^{7}$ Furthermore, we showed that long-term treatment of FDR with an insulin-sensitizing drug, pioglitazone, normalized not only insulin resistance and hyperinsulinemia but also hypertension and altered neurogenic vascular responses. ${ }^{8}$ Thus, we have proposed that the insulin resistance associated with hyperinsulinemia elicits abnormal neuronal regulation of vascular tone, which contributes in part to the development of hypertension. However, the cause of hypertension associated with insulin resistance and/or hyperinsulinemia is still unclear.

${ }^{1}$ Department of Clinical Pharmaceutical Science, Graduate School of Medicine, Dentistry and Pharmaceutical Sciences, Okayama University, Okayama, Japan; ${ }^{2}$ Department of Molecular Design for Medicine, Graduate School of Medicine, Dentistry and Pharmaceutical Sciences, Okayama University, Okayama, Japan; ${ }^{3}$ Department of life Science, Okayama University of Science, Okayama, Japan and ${ }^{4}$ Department of Pharmaceutical Care and Health Science, Graduate School of Medicine, Dentistry and Pharmaceutical Sciences, Okayama University, Okayama, Japan

Correspondence: Dr S Takatori, Department of Clinical Pharmaceutical Science, Graduate School of Medicine, Dentistry and Pharmaceutical Sciences, Okayama University, 1-1-1 Tsushima-naka, Okayama 700-8530, Japan.

E-mail: s.takatori@pharm.okayama-u.ac.jp

Received 27 October 2010; revised 25 February 2011; accepted 5 May 2011; published online 28 July 2011 
It is widely accepted that systemic blood pressure is controlled and maintained by changing vascular resistance via neuronal and hormonal factors. Also, large vascular beds such as the mesenteric vascular bed contribute to the maintenance of the blood pressure. ${ }^{9}$ It has been demonstrated that rat mesenteric resistance arteries are densely innervated by both sympathetic adrenergic nerves and capsaicinsensitive CGRPergic nerves. ${ }^{10}$ Moreover, the decreased CGRPergic vasodilator nerve innervation in mesenteric resistance arteries has been shown to contribute to the development and maintenance of hypertension in spontaneously hypertensive rats. ${ }^{11-14}$

Thus, in the present study, to further clarify mechanisms underlying altered neuronal vascular control in chronic hyperinsulinemia, we investigated the adrenergic and CGRPergic nerve-mediated vascular responses to periarterial nerve stimulation (PNS) in perfused mesenteric vascular beds of FDR. Additionally, we examined the distribution of sympathetic adrenergic nerves and CGRPergic nerves in mesenteric arteries using immunohistochemical techniques.

\section{METHODS}

Animals

Thirty-six male Wistar rats weighing $200-250$ g were used in this study. The animals were given food and water ad libitum. They were housed at the Animal Research Center of Okayama University at a controlled ambient temperature of $22 \pm 2{ }^{\circ} \mathrm{C}$ with $50 \pm 10 \%$ relative humidity and a 12 -h light/12-h dark cycle (lights on at $0800 \mathrm{~h}$ ). This study was carried out in accordance with the Guidelines for Animal Experiments at Okayama University Advanced Science Research Center, Japanese Government Animal Protection and Management Law (No. 105) and Japanese Government Notification on Feeding and Safekeeping of Animals (No. 6). Every effort was made to minimize the number of animals used and their suffering.

\section{Fructose-induced hyperinsulinemia in Wistar rats}

At 6 weeks of age, the animals were randomly divided into two groups: one group received normal chow and water and another group received normal chow and $15 \%$ fructose solution for 10 weeks. Body weight and food and liquid intake were measured at 6 and 16 weeks of age.

\section{Biochemical analysis}

At 6 and 16 weeks of age, blood samples were obtained by cardiopuncture after $12 \mathrm{~h}$ fasting. The plasma glucose level was measured using a glucose analyzer (ADVANTAGE; Boehringer Mannheim, Tokyo, Japan). Plasma insulin was measured by a double-antibody method with an enzyme-linked immunosorbent assay (ELISA) insulin kit (Morinaga Biochemistry, Kanagawa, Japan). The glucose-insulin index was calculated as the product of the glucose and insulin areas under the curve during a $0.3 \mathrm{~g} \mathrm{~kg}^{-1}$ intravenous glucose tolerance test. $^{15}$

\section{Systolic blood pressure and heart rate measurements}

Systolic blood pressure and heart rate under the conscious state were measured with a tail-cuff plethysmograph (TK-370C, Unicom, Tokyo, Japan) between 0900 and $1200 \mathrm{~h}$ at 6 and 16 weeks of age. The average of five readings was used.

\section{Perfusion of the mesenteric vascular beds}

The animals were anesthetized with pentobarbital- $\mathrm{Na}\left(50 \mathrm{mg} \mathrm{kg}^{-1}\right.$, intraperitoneally) and the mesenteric vascular beds were isolated and prepared for perfusion as described previously. ${ }^{10}$ The superior mesenteric artery was cannulated and flushed gently with Krebs-Ringer bicarbonate solution (Krebs solution) to eliminate blood in the vascular bed. After removal of the entire intestine and associated vascular bed, the mesenteric vascular bed was separated from the intestine by cutting close to the intestinal wall. Only four main arterial branches from the superior mesenteric trunk running to the terminal ileum were perfused. All other branches of the superior mesenteric artery were tied off. The isolated mesenteric vascular bed was then placed in a water-jacketed organ bath maintained at $37^{\circ} \mathrm{C}$ and perfused with a modified (see below) Krebs solution at a constant flow rate of $5 \mathrm{ml}$ per min with a peristaltic pump (model AC-2120; ATTO, Tokyo, Japan). The preparation was also superfused with the same solution at a rate of $0.5 \mathrm{ml}$ per min to prevent drying. The Krebs solution was bubbled with a mixture of $95 \% \mathrm{O}_{2}$ and $5 \% \mathrm{CO}_{2}$ before passage through a warming coil maintained at $37^{\circ} \mathrm{C}$. The modified Krebs solution had the following composition (mM): $\mathrm{NaCl} 119.0 ; \mathrm{KCl} 4.7 ; \mathrm{CaCl}_{2} 2.4 ; \mathrm{MgSO}_{4}$ 1.2; $\mathrm{NaHCO}_{3}$ 25.0; $\mathrm{KH}_{2} \mathrm{PO}_{4}$ 1.2; disodium EDTA 0.03 and dextrose 11.1 (pH 7.4). Changes in the perfusion pressure were measured with a pressure transducer (model TP-400T; Nihon Kohden, Tokyo, Japan) and recorded using a pen recorder (model U-228; Nippon Denshi Kagaku, Tokyo, Japan).

\section{Experimental protocol for vascular response measurements}

After 30 min perfusion, isolated mesenteric vascular beds with resting tone were subjected to PNS $(4,8$ and $12 \mathrm{~Hz})$ and a bolus injection of norepinephrine (NE) $(5$ and $10 \mathrm{nmol})$, which induced an increase in perfusion pressure due to vasoconstriction. Thereafter, Krebs solution was switched to Krebs solution containing $5 \mu \mathrm{M}$ guanethidine (adrenergic neuron blocker) and $7 \mu \mathrm{M}$ methoxamine ( $\alpha 1$-adrenergic receptor agonist) to increase the perfusion pressure to $\sim 100 \mathrm{~mm} \mathrm{Hg}$ levels. When methoxamine-induced perfusion pressure increase was $<30 \mathrm{~mm} \mathrm{Hg}$, preparations were eliminated from data. After the elevated perfusion pressure stabilized, PNS $(1,2$ and $4 \mathrm{~Hz})$ and bolus injections of CGRP (50 and $100 \mathrm{pmol}$ ) were applied to induce a reduction in the perfusion pressure due to vasodilation. PNS was applied via bipolar platinum ring electrodes placed around the superior mesenteric artery. Rectangular pulses of $1 \mathrm{~ms}$ and a supramaximal voltage $(50 \mathrm{~V})$ were applied for $30 \mathrm{~s}$ via an electronic stimulator (model SEN 3301; Nihon Kohden, Tokyo, Japan). NE and CGRP were directly injected into the perfusate proximal to the arterial cannula with an infusion pump (model 975; Harvard Apparatus, Holliston, MA, USA), with perfusion pressure returning to baseline values after the respective neurogenic responses. A volume of $100 \mu \mathrm{l}$ was injected over a period of $12 \mathrm{~s}$. At the end of each experiment, $100 \mu \mathrm{m}$ papaverine was perfused to produce complete relaxation. Vasoconstriction was expressed as the peak increase in mean perfusion pressure $(\Delta \mathrm{mm} \mathrm{Hg})$ from the baseline value before PNS or NE injection. Vasodilation was expressed as the percentage of maximum relaxation induced by perfusion of papaverine.

\section{Immunohistochemical study}

The animals were anesthetized with pentobarbital-Na $\left(50 \mathrm{mg} \mathrm{kg}^{-1}\right.$, intraperitoneally). The superior mesenteric artery was cannulated with polyethylene tubing and infused with Zamboni solution (2\% paraformaldehyde and 15\% picric acid in $0.15 \mathrm{M}$ phosphate buffer) and the mesenteric artery was removed together with the intestine as described previously. ${ }^{14}$ The third branch of the mesenteric artery proximal to the intestine was removed and immersion fixed in the Zamboni solution for $48 \mathrm{~h}$. After fixation, the artery was repeatedly rinsed with phosphate-buffered saline and immersed in phosphate-buffered saline containing $0.5 \%$ Triton X-100 overnight, and then incubated with phosphate-buffered saline containing normal goat serum (1:100) for $60 \mathrm{~min}$. The artery was then incubated with a rabbit polyclonal anti-CGRP (Biogenesis, Poole, UK) antibody at a dilution of 1:300 or a rabbit polyclonal antineuropeptide Y (NPY) (Phoenix Pharmaceuticals, Belmont, CA, USA) antibody at a dilution of $1: 300$ for $72 \mathrm{~h}$ at $4{ }^{\circ} \mathrm{C}$. After the incubation, the site of the antigen-antibody reaction was revealed by incubation with fluorescein-5isothiocyanate-labeled goat anti-rabbit IgG (diluted 1:100) (ICN Pharmaceuticals, Aurora, OH, USA) for $60 \mathrm{~min}$. Thereafter, the artery was mounted on slides, coverslipped with glycerol/phosphate-buffered saline $(2: 1 \mathrm{v} / \mathrm{v})$ and observed under a confocal laser scanning microscope (CLSM510, Carl Zeiss $\mathrm{GmbH}$, Jena, Germany) in the Okayama University Medical School Central Research Laboratory.

\section{Immunohistochemical analysis}

The immunostaining density of CGRP-like immunoreactive (CGRP-LI) or NPY-like immunoreactive (LI) nerve fibers was analyzed using the method as described by Hobara et al. ${ }^{11}$ Since the fluorescence intensity differed depending on the day of the experiment, the mesenteric arteries were isolated, fixed and immunostained at the same time on the same day and mounted on the same slide glass. Age-matched control rats were used as a control for the intensity in 
each experiment. For the quantitative evaluation of NPY-LI and CGRP-LI, confocal projection images of NPY and CGRP immunostaining, which were patched together with $8-10$ overlapping images $(0.1 \mu \mathrm{m}$ scanning), were magnified at $\times 20$ and digitized as Tagged Image File Format (TIFF) images using a digital camera system (Olympus SP-1000, Olympus, Tokyo, Japan) and imported into a Windows XP computer (Toshiba, Tokyo, Japan). The stored digital images were analyzed using image-processing software (Simple PCI; Compix, Imaging Systems, Cranberry Township, PA, USA). The extraction of specific color and measured field commands was used to extract the NPY-LI and CGRP-LI areas (which were stained green). Extraction of the signal was carried out using specific protocols based on the hue, lightness and saturation color parameters. A measured field of $100 \mu \mathrm{m} \times 100 \mu \mathrm{m}\left(10000 \mu \mathrm{m}^{2}\right)$, which contained the adventitia layer including immunostained perivascular nerve fibers, was randomly selected on magnified images of the whole mount artery. The objective areas command was used to calculate the percentages of NPY-LIand CGRP-LI-positive areas. The intensity of staining was estimated using a point counting computer program and the background level was subtracted from the experimental value to yield the corrected intensity. The average density in three arteries was taken as the nerve density per animal. To determine the number of CGRP-LI and NPY-LI fibers, five horizontal lines were drawn on the image of the blood vessel in the same region where the density was estimated by computer analysis. Then, the number of fibers that crossed each line was counted and the average number in three arteries was taken as the total number of fibers per animal. Because immunostaining of NPY-LI and CGRP-LI fibers in some preparations showed weak fluorescent staining due to weak antibody absorption, six of nine data were selected as data.

\section{Plasma NE measurements}

At 16 weeks of age, blood samples obtained from the jugular vein were centrifuged immediately and the plasma was stored at $-80^{\circ} \mathrm{C}$ until assay. $\mathrm{NE}$ in the plasma was absorbed onto $5 \mathrm{mg}$ alumina, and the extract obtained with $50 \mu \mathrm{l}$ of $2 \%$ acetic acid was assayed by high-performance liquid chromatography with electrochemical detection (model HTEC-500; EICOM, Kyoto, Japan). 3,4-dihydroxybenzylamine hydrobromide (Sigma-Aldrich Japan, Tokyo, Japan) was used as the internal standard.

\section{Measurements of CGRP in dorsal root ganglia}

At 16 weeks of age, under pentobarbital-Na anesthesia, the thoracic and lumbar dorsal root ganglia (DRG) from control and FDR were immediately dissected, frozen in liquid $\mathrm{N}_{2}$ and stored at $-80^{\circ} \mathrm{C}$ until subsequent analysis of CGRP content. The DRG was homogenized with scissors in $300 \mu \mathrm{l}$ of Tris-buffered saline (20 mM Tris- $\mathrm{HCl}$ (pH 7.4), $1 \mathrm{~mm}$ EDTA) containing a protease inhibitor cocktail (Biovision Research Products, Mountain View, CA, USA). The homogenate was centrifuged at $600 \mathrm{~g}$ for $10 \mathrm{~min}$ at $4{ }^{\circ} \mathrm{C}$. The supernatant was further centrifuged at $100000 \mathrm{~g}$ for $1 \mathrm{~h}$ at $4{ }^{\circ} \mathrm{C}$. The resulting pellet was then washed and resuspended with the same Tris-buffered saline with $0.1 \%$ Triton X-100. The solution was centrifuged at $100000 \mathrm{~g}$ for $1 \mathrm{~h}$ at $4{ }^{\circ} \mathrm{C}$. The concentration of CGRP in DRG was determined using an ELISA for CGRP kit (Bertin Pharma, Bordeaux, France).

\section{Statistical analysis}

Experimental results are expressed as the mean \pm s.e.m. Statistical analysis was performed using the Student's unpaired $t$-test or one-way analysis of variance followed by Tukey's test, as appropriate. Correlation analysis was carried out using Pearson's correlation test. A $P$-value $<0.05$ was considered statistically significant.

\section{RESULTS}

\section{Changes in body weight, food and fluid intakes}

There was no significant difference in body weight between FDR and control rats at 6 and 16 weeks of age (Table 1). After starting 15\% fructose solution as drinking fluid, food intake in FDR was markedly decreased compared with that in control rats, but fluid intake in FDR was significantly increased compared with that of control rats (Table 1).
Table 1 Changes in body weight, food and fluid intake, blood glucose level, plasma insulin, glucose-insulin index, systolic blood pressure and heart rate in $15 \%$ fructose-drinking rats (FDR; $n=9$ ) and waterdrinking rats (control rats; $n=9$ ) between 6 and 16 weeks of age

\begin{tabular}{lcc}
\hline & 6 weeks & 16 weeks \\
\hline $\begin{array}{lll}\text { Body weight } \\
\text { Control }\end{array}$ & \\
FDR & $195.2 \pm 11.1$ & $435.0 \pm 11.5$ \\
& $194.0 \pm 13.1$ & $433.3 \pm 13.2$
\end{tabular}

Food intake (g per $100 \mathrm{~g}$ BW per day)

$\begin{array}{lll}\text { Control } & 7.3 \pm 0.4 & 4.9 \pm 0.2 \\ \text { FDR } & 6.5 \pm 0.7 & 2.6 \pm 0.2^{* *}\end{array}$

Fluid intake (g per $100 \mathrm{~g}$ BW per day)

Control $26.5 \pm 1.5 \quad 11.6 \pm 1.3$

$\begin{array}{lll}\text { FDR } & 24.1 \pm 0.7 & 15.4 \pm 1.5^{*}\end{array}$

Plasma glucose $\left(\mathrm{mg} \mathrm{dl}^{-1}\right)$

$\begin{array}{lll}\text { Control } & 90.2 \pm 5.5 & 119.3 \pm 2.5\end{array}$

$\begin{array}{lll}\text { FDR } & 94.5 \pm 3.9 & 107.6 \pm 7.2\end{array}$

Plasma insulin $\left(\mu \cup \mathrm{mr}^{-1}\right)$

$\begin{array}{lll}\text { Control } & 12.9 \pm 5.4 & 12.9 \pm 3.8\end{array}$

$\begin{array}{lll}\text { FDR } & 11.7 \pm 3.3 & 28.4 \pm 8.4^{* *}\end{array}$

Glucose-insulin index (units $\times 10^{6}$ )

$\begin{array}{lrr}\text { Control } & 37.2 \pm 3.1 & 48.0 \pm 9.2\end{array}$

$\begin{array}{lll}\text { FDR } & 34.5 \pm 1.2 & 171.2 \pm 6.0^{* *}\end{array}$

Systolic blood pressure $(\mathrm{mm} \mathrm{Hg})$

Control $\quad 92.6 \pm 3.1 \quad 109.6 \pm 2.3$

$\begin{array}{lll}\text { FDR } & 91.9 \pm 2.3 & 132.3 \pm 4.5^{* *}\end{array}$

Heart rate (beats per min)

$\begin{array}{lll}\text { Control } & 406.0 \pm 8.3 & 348.3 \pm 11.9\end{array}$

$\begin{array}{lll}\text { FDR } & 388.8 \pm 8.6 & 369.6 \pm 12.0\end{array}$

Values are expressed as the mean \pm s.e.m.

${ }^{*} P<0.05$.

${ }^{* *} P<0.01$ vs. control rats.

Changes in blood glucose levels, plasma insulin and the glucoseinsulin index

As shown in Table 1, there was no significant difference in blood glucose level between FDR and control rats at 16 weeks of age. However, at 16 weeks of age, the plasma insulin level in FDR was significantly higher than that in control rats (Table 1). Additionally, the glucose-insulin index in FDR, which is the insulin resistance index, was significantly higher than that in control rats at 16 weeks of age (Table 1).

\section{Changes in systolic blood pressure and heart rate}

As shown in Table 1, systolic blood pressure in FDR was elevated by $15 \%$ fructose solution treatment for 10 weeks. Significant differences in systolic blood pressure between FDR and control rats were found at 16 weeks of age (Table 1). However, there was no significant difference in heart rate between FDR and control rats throughout the experimental period (Table 1).

\section{Changes in vasoconstrictor responses to PNS and bolus injections} of NE

As shown in Figures 1a and c, PNS at 4, 8 and $12 \mathrm{~Hz}$ in the perfused mesenteric vascular beds with resting tone produced a frequency- 

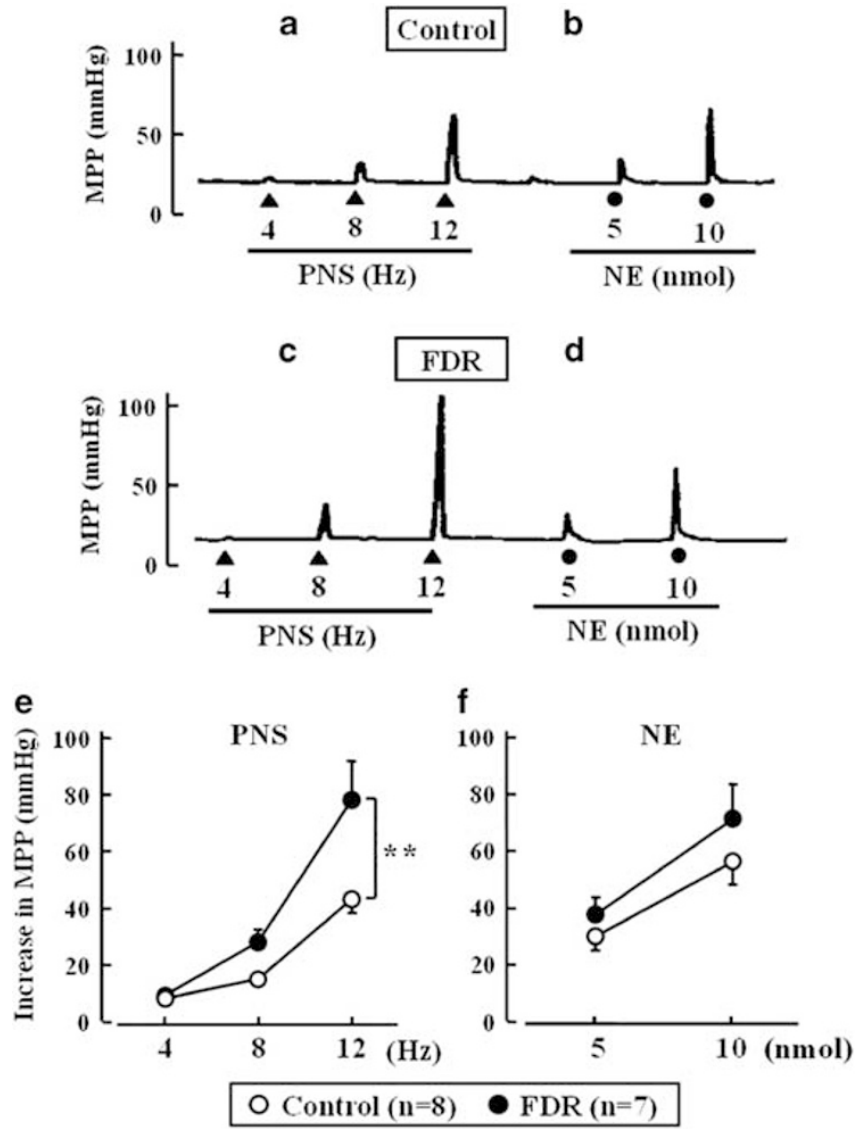

Figure 1 Typical records (a-d) and line graphs (e, f) showing vasoconstrictor responses induced by PNS and bolus infusion of NE ( 5 and $10 \mathrm{nmol}$ ) in perfused mesenteric vascular beds isolated from control rats and 15\% fructose-drinking rats (FDR) at 16 weeks of age. Vasoconstrictor responses in the preparation with resting tone. $\mathrm{O}$, Control rats; $\bullet$, FDR. Each bar indicates the mean \pm s.e.m. of $7-8$ experiments. ${ }^{*} P<0.01$ vs. control rats. MPP, mean perfusion pressure; PPV, perfusion of papaverine.

dependent increase in perfusion pressure due to vasoconstriction. The PNS-induced vasoconstriction was abolished by prazosin ( $\alpha 1$-adrenoceptor antagonist) and guanethidine (adrenergic neuron blocker) (data not shown), indicating that the response is mediated by NE released from the stimulation of periarterial adrenergic nerves. As shown in Figures $1 \mathrm{~b}$ and d, bolus injections of NE at concentrations of 5 and $10 \mathrm{nmol}$ also caused concentration-dependent vasoconstriction, which was blocked by prazosin, but not by guanethidine (data not shown), indicating that the response was mediated by the stimulation of postsynaptic $\alpha 1$-adrenoceptors. As shown in Figure 1e, the vasoconstrictor response to PNS in FDR at 16 weeks old was significantly greater than that in age-matched control rats. However, there was no significant difference in vasoconstrictor responses to bolus injections of NE between control rats and FDR (Figure 1f).

\section{Changes in plasma NE}

As previously mentioned, the PNS-induced vasoconstriction is mediated by NE released from the stimulation of periarterial adrenergic nerves. ${ }^{10}$ In addition to the neurogenic vascular responsiveness in the perfused mesenteric vascular beds, to clarify the effect of insulin resistance and chronic hyperinsulinemia on sympathetic adrenergic activity, we investigated the plasma NE levels in control rats and FDR by high-performance liquid chromatography analysis. As a result, the
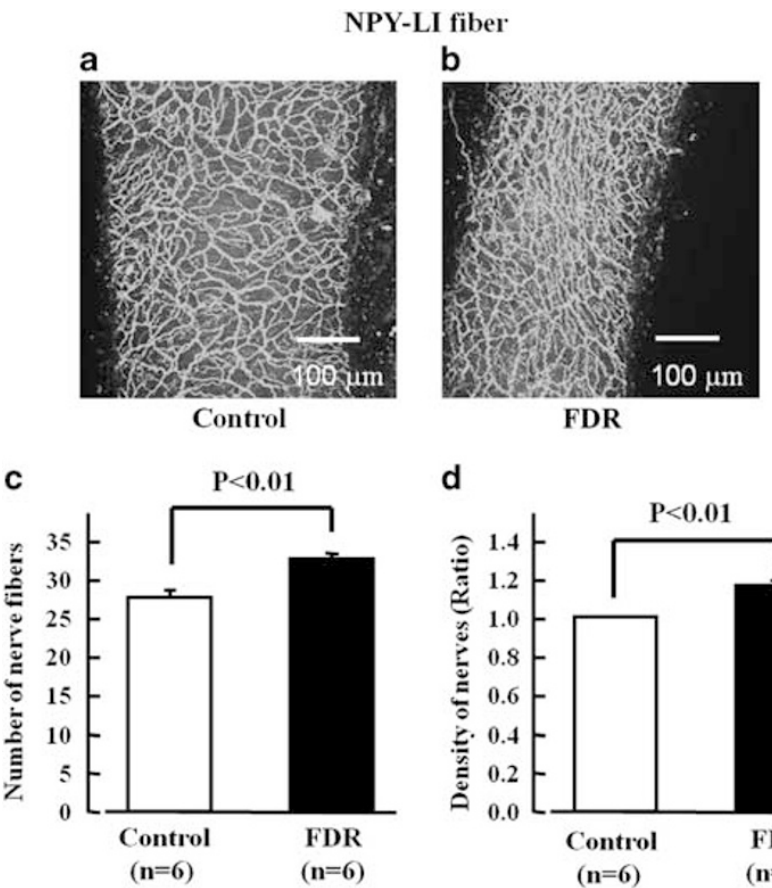

d

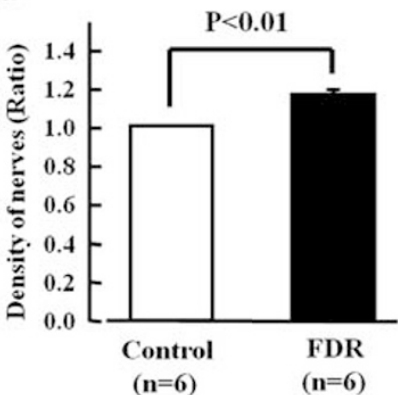

Figure 2 Typical images (a, b) and bar graph (c, d) showing number and density of neuropeptide $Y$ (NPY)-like immunoreactivity (LI)-containing nerve fibers in distal mesenteric isolated from control rats and $15 \%$ fructosedrinking rats (FDR) at 16 weeks of age. The scale bar $(100 \mu \mathrm{m})$ in the right lower corner of each image. Each bar indicates the mean \pm s.e.m. of 6 experiments.

plasma NE levels in FDR of 16 weeks old $\left(148.3 \pm 2.7 \mathrm{pg} \mathrm{ml}^{-1}\right.$, $P<0.05)$ were significantly higher than those in age-matched control rats $\left(85.0 \pm 1.9 \mathrm{pg} \mathrm{ml}^{-1}\right)$.

\section{Changes in distribution of sympathetic NPY-LI nerve fibers} in mesenteric arteries

The distal small mesenteric artery from control rats and FDR at 16 weeks of age had dense innervation of NPY-LI nerve fibers (Figures 2a and $b$ ). As shown in Figures $2 c$ and $d$, the density, which was quantified by computer-assisted image processing, and numbers, which were visually counted, in NPY-LI nerve fibers of FDR, were significantly greater than those in age-matched control rats. There were significant positive correlations between the density and numbers of NPY-LI nerve fibers in control rats $\left(P<0.05, R^{2}=0.8223\right)$ and FDR $\left(P<0.05, R^{2}=0.9131\right)$.

\section{Changes in vasodilator responses to PNS and bolus injections of CGRP}

To maintain the active tone of the mesenteric artery, the preparation was contracted by continuous perfusion of $7 \mu \mathrm{m}$ methoxamine $(\alpha 1$ adrenergic receptor agonist) in the presence of $5 \mu \mathrm{M}$ guanethidine (adrenergic neuron blocker). In this preparation, PNS at 1, 2 and $4 \mathrm{~Hz}$ caused a frequency-dependent decrease in perfusion pressure due to vasodilation (Figures $3 \mathrm{a}$ and $\mathrm{c}$ ). The vasodilator response to PNS has been shown to be mediated by CGRPergic nerves, since the response was blocked by a CGRP receptor antagonist (CGRP (8-37)) and a CGRP depletor (capsaicin). ${ }^{16,17}$ Bolus injections of CGRP at concentrations of 50 and $100 \mathrm{pmol}$ also induced concentration-dependent vasodilation (Figures $3 \mathrm{~b}$ and $\mathrm{d}$ ), which has been shown to be mediated by postsynaptic CGRP receptors. ${ }^{18}$ 

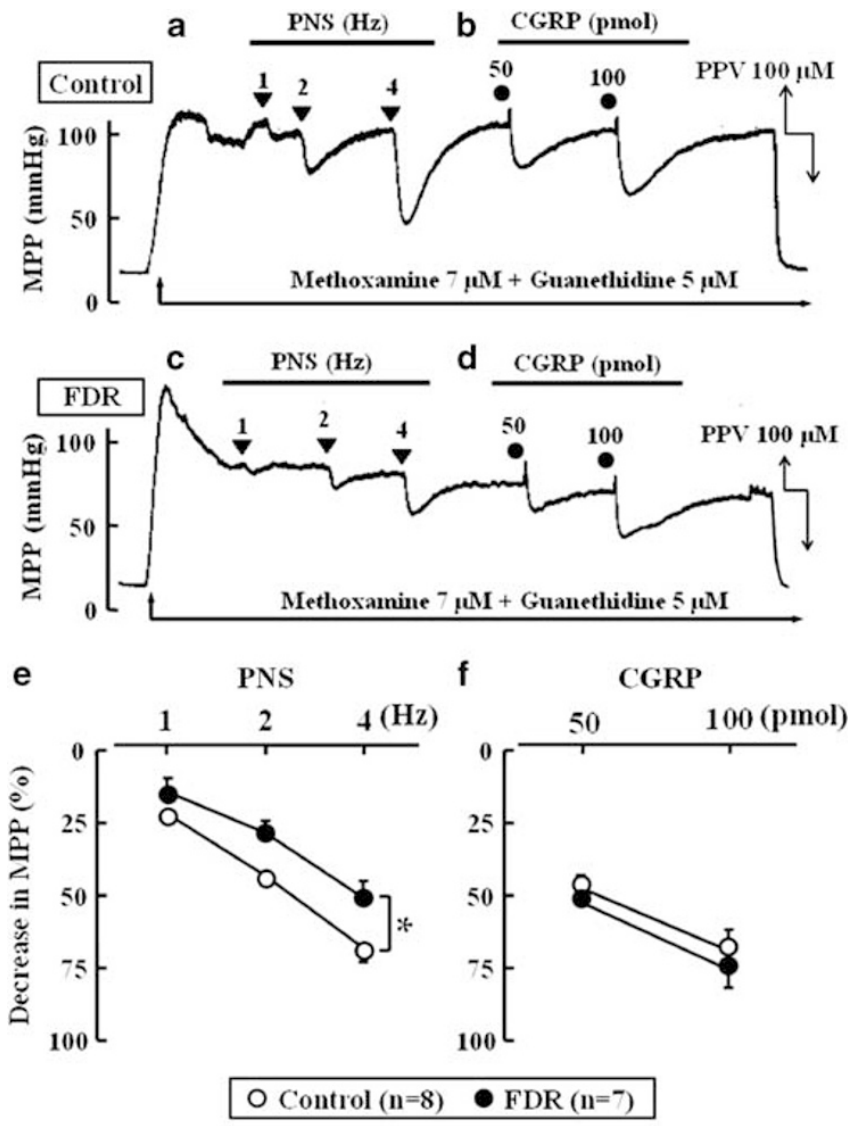

Figure 3 Typical records (a-d) and line graphs (e, f) showing vasodilator responses induced by PNS and bolus infusion of CGRP (50 and 100 pmol) in perfused mesenteric vascular beds isolated from control rats and $15 \%$ fructose-drinking rats (FDR) at 16 weeks of age. Vasodilator responses in the preparation with active tone produced by $7 \mu \mathrm{m}$ methoxamine in the presence of $5 \mu \mathrm{m}$ guanethidine. $\bigcirc$, Control rats; $\bullet$, FDR. Each bar indicates the mean \pm s.e.m. of $7-8$ experiments. ${ }^{*} P<0.05$ vs. control rats. MPP, mean perfusion pressure; PPV, perfusion of papaverine.

Additionally, there was no significant difference in the methoxamine-induced rises in mean perfusion pressure before PNS between control rats $(103.1 \pm 8.4 \mathrm{~mm} \mathrm{Hg}, n=8)$ and FDR $(94.7 \pm 12.9 \mathrm{~mm} \mathrm{Hg}$, $n=7)$. The PNS-induced vasodilator responses in FDR preparations were significantly smaller than those in age-matched control preparations (Figure 3e). However, no significant difference in exogenous CGRP-induced vasodilation was found between control and FDR preparations (Figure 3f).

\section{Changes in CGRP content in the DRG}

As previously mentioned, we demonstrated that the PNS-induced vasodilator response is mediated by CGRP released from CGRPergic nerve terminals. ${ }^{16,17}$ A prominent site of CGRP synthesis has been shown to be the DRG, which contains the cell bodies of primary afferent neurons that extend CGRPergic nerves to peripheral sites such as blood vessels. ${ }^{19}$ In addition to neurogenic vascular responsiveness in the perfused mesenteric vascular beds, to clarify the effect of insulin resistance and chronic hyperinsulinemia on CGRPergic nerves activity, we investigated the content of CGRP in DRG isolated from control rats and FDR by an ELISA. Consequently, CGRP content in FDR $\left(248.9 \pm 25.1 \mathrm{pg} \mathrm{ml}^{-1}, P<0.05\right)$ was significantly smaller than that in age-matched control rats $\left(358.4 \pm 27.0 \mathrm{pg} \mathrm{ml}^{-1}\right)$.

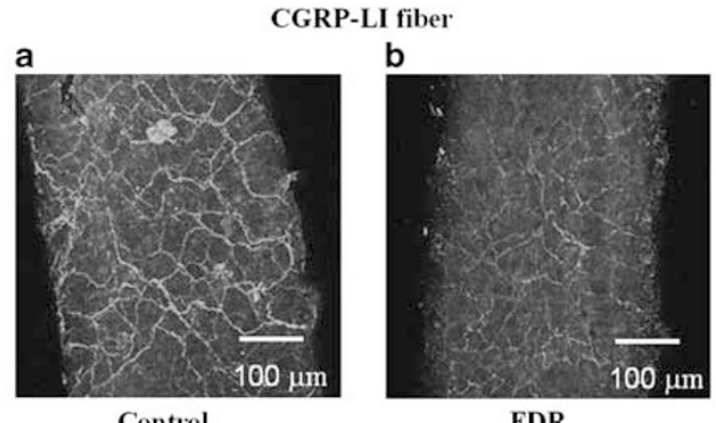

FDR

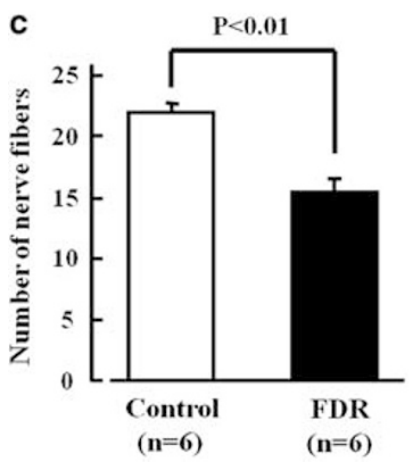

d

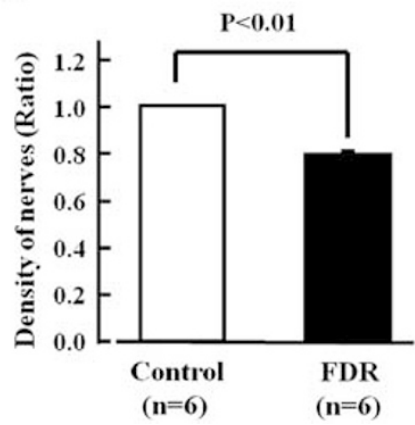

Figure 4 Typical images (a, b) and bar graph (c, d) showing number and density of calcitonin gene-related peptide (CGRP)-like immunoreactivity (LI)containing nerve fibers in distal mesenteric isolated from control rats and $15 \%$ fructose-drinking rats (FDR) at 16 weeks of age. The scale bar $(100 \mu \mathrm{m})$ in the right lower corner of each image. Each bar indicates the mean \pm s.e.m. of 6 experiments.

Changes in distribution of CGRP-LI nerve fibers in mesenteric arteries

As shown in typical images of Figures $4 \mathrm{a}$ and $\mathrm{b}$, the mesenteric artery of control rats and FDR at 16 weeks of age had dense innervation of CGRP-LI nerve fibers. The density and numbers of CGRP-LI nerve fibers in FDR at 16 weeks of age were significantly smaller than those in age-matched control rats (Figures $4 \mathrm{c}$ and $\mathrm{d}$ ).

There were significant positive correlations between the density and numbers of CGRP-LI nerve fibers in control rats $(P<0.05$, $\left.R^{2}=0.8021\right)$ and FDR $\left(P<0.05, R^{2}=0.9465\right)$.

\section{DISCUSSION}

In the present study, FDR given 15\% fructose solution as their drinking water showed a two-fold increase in plasma levels of insulin and a 3.5-fold increase in the glucose-insulin index without a significant change in blood glucose levels, suggesting that these animals had hyperinsulinemia due to insulin resistance. Additionally, it is important to note that FDR with hyperinsulinemia also had hypertension. These findings are in good accordance with previous reports. ${ }^{7,8,20}$ Therefore, hyperinsulinemia based on insulin resistance is likely to be associated with hypertension.

The present study demonstrated that the vasoconstrictor response to PNS in the mesenteric vascular beds of FDR was significantly greater than that in control rats. The PNS-induced vasoconstrictor response in the rat mesenteric arteries has been shown to be mediated by activation of sympathetic adrenergic nerves, since a neurotoxin (tetrodotoxin), an adrenergic neuron blocker (guanethidine), an adrenergic neurotoxin (6-hydroxydopamine) and an $\alpha 1$-adrenoceptor antagonist (prazosin) abolished the response. ${ }^{21-23}$ 
On the other hand, FDR showed an unchanged vasoconstrictor response to exogenously applied NE, which is mediated by the postsynaptic $\alpha 1$-adrenoceptor. Taken together, the present findings strongly suggest that neurotransmitter NE release from sympathetic nerve terminals is facilitated by enhanced sympathetic nerve activity in a hyperinsulinemic state without affecting sensitivity of postsynaptic $\alpha 1$-adrenoceptors. This notion is supported by the present finding that serum NE levels in FDR, which indicate sympathetic adrenergic activity, were significantly greater than those in the control.

The pattern of distribution of NPY, which coexists with NE in the nerves, has been shown to be the same as that of sympathetic adrenergic nerves. ${ }^{24}$ The present findings showed that the density of adrenergic NPY-LI fiber innervation in FDR was significantly increased when compared with control rats. Thus, it is likely that the enhanced adrenergic nerve-mediated vasoconstriction in mesenteric arteries of FDR mainly results from increased density of sympathetic adrenergic innervation.

Moreover, another important consideration of our findings is that the vasodilator response to PNS in the mesenteric arteries of FDR was decreased without affecting vasodilator response to exogenously applied CGRP when compared with control rats. In the rat perfused mesenteric vascular bed precontracted with methoxamine in the presence of guanethidine, the vasodilator response to PNS has been shown to be mediated by the CGRP-containing vasodilator nerve, as the response is blocked by CGRP (8-37), a CGRP receptor antagonist, and capsaicin, which causes CGRP depletion in CGRPergic nerves. ${ }^{16,17}$ It seems likely that CGRPergic nerve activity is decreased in mesenteric arteries of FDR. This notion is supported by the present finding that the CGRP content in the DRG of FDR was significantly lower than that in control rats, since the DRG, which contains the cell bodies of CGRPergic nerves, is a prominent site of CGRP synthesis. The synthesized CGRP is transported to peripheral sites such as blood vessels and the spinal cord via axonal flow. Therefore, it appears that transmitter (CGRP) release from CGRPergic nerves in the mesenteric artery is decreased in FDR.

Previous in vitro studies using rat mesenteric resistance arteries have demonstrated that CGRPergic vasodilator nerves functionally attenuate adrenergic nerve-mediated vasoconstriction, suggesting that adrenergic and CGRPergic nerves regulate the tone of resistance arteries by reciprocal interactions. ${ }^{25}$ In our in vitro study, the PNS-induced pressor response in the mesenteric blood vessels resulted from activation of both sympathetic nerves and CGRPergic nerves. Therefore, it seems likely that the increase in the vasoconstrictor response to PNS observed in the FDR resulted in part from the decreased vasodilation mediated by CGRPergic nerves.

It should be noted that FDR showed a decrease in the density of CGRP-LI fiber in the mesenteric blood vessels. Therefore, it is very likely that the reduced density of CGRPergic nerve fibers is responsible for the reduction in CGRPergic nerve-mediated vasodilation in FDR. Many reports demonstrated that CGRPergic nerves were highly dependent on neurotrophic support provided by insulin. ${ }^{26,27}$ Based on these evidences, decrease in the density of CGRPergic nerve fibers in FDR mesenteric arteries might be involved in impaired insulin action on CGRPergic nerves, although further investigations are needed to test this mechanism. In contrast, previous reports demonstrate that sympathetic nerve terminals were less susceptible to insulin resistance than other organs in insulin-resistant model. ${ }^{28}$ Furthermore, in vitro studies showed that physiological concentrations of insulin stimulate neurite outgrowth in sympathetic nerves. ${ }^{29-31}$ Thus, it is likely that increased density of sympathetic adrenergic nerves in
FDR mesenteric arteries is caused by hyperactivity of excess insulin on sympathetic nerves.

In conclusion, the present findings suggest that the hyperinsulinemia based on insulin resistance in FDR produces remodeling of perivascular innervation, leading to enhanced sympathetic adrenergic nerve activity and decreased CGRPergic nerve activity. Therefore, it is likely that dysfunction of the neuronal vascular control system resulting from abnormal innervation of perivascular nerves in the mesenteric blood vessels induced by the hyperinsulinemic state and insulin resistance may be responsible for the development of hypertension in FDR.

\section{CONFLICT OF INTEREST}

The authors declare no conflict of interest.

1 Skarfors ET, Selinus KI, Lithell HO. Risk factors for developing non-insulin dependent diabetes: a 10 year follow up of men in Uppsala. BMJ 1991; 303: 755-760.

2 Haffner SM, Valdez RA, Hazuda HP, Mitchell BD, Morales PA, Stern MP. Prospective analysis of the insulin-resistance syndrome (syndrome X). Diabetes 1992; 41: 715-722.

3 Lissner L, Bengtsson C, Lapidus L, Kristjansson K, Wedel H. Fasting insulin in relation to subsequent blood pressure changes and hypertension in women. Hypertension 1992; 20: 797-801.

4 Modan M, Halkin H, Almog S, Lusky A, Eshkol A, Shefi M, Shitrit A, Fuchs Z. Hyperinsulinemia. A link between hypertension obesity and glucose intolerance. J Clin Invest 1985; 75: 809-817.

5 Landsberg L. Diet, obesity and hypertension: an hypothesis involving insulin, the sympathetic nervous system, and adaptive thermogenesis. Q J Med 1986; 61: 1081-1090.

6 Reaven GM, Hoffman BB. A role for insulin in the aetiology and course of hypertension? Lancet 1987; 2: 435-437.

7 Takatori S, Zamami Y, Mio M, Kurosaki Y, Kawasaki H. Chronic hyperinsulinemia enhances adrenergic vasoconstriction and decreases calcitonin gene-related peptidecontaining nerve-mediated vasodilation in pithed rats. Hypertens Res 2006; 29: 361-368.

8 Takatori S, Zamami Y, Yabumae N, Hanafusa N, Mio M, Egawa T, Kawasaki H. Pioglitazone opposes neurogenic vascular dysfunction associated with chronic hyperinsulinaemia. Br J Pharmacol 2008; 153: 1388-1398.

9 Lundgren 0 . Role of splanchnic resistance vessels in overall cardiovascular homeostasis. Fed Proc 1983; 42: 1673-1677.

10 Kawasaki H, Takasaki K, Saito A, Goto K. Calcitonin gene-related peptide acts as a novel vasodilator neurotransmitter in mesenteric resistance vessels of the rat. Nature 1988; 335: 164-167.

11 Hobara N, Gessei-Tsutsumi N, Goda M, Takayama F, Akiyama S, Kurosaki Y, Kawasaki $\mathrm{H}$. Long-term inhibition of angiotensin prevents reduction of periarterial innervation of calcitonin gene-related peptide (CGRP)-containing nerves in spontaneously hypertensive rats. Hypertens Res 2005; 28: 465-474.

12 Kawasaki $\mathrm{H}$, Nuki $\mathrm{Y}$, Yamaga N, Kurosaki Y, Taguchi T. Decreased depressor response mediated by calcitonin gene-related peptide (CGRP)-containing vasodilator nerves to spinal cord stimulation and levels of CGRP MRNA of the dorsal root ganglia in spontaneously hypertensive rats. Hypertens Res 2000; 23 : 693-699.

13 Kawasaki H. Effects of chronic administration of antihypertensive drugs on vasodilation mediated by calcitonin gene-related peptide-containing vasodilator nerves in spontaneously hypertensive rats. Clin Exp Pharmacol Physiol 1992; 19: 569-573.

14 Kawasaki H, Saito A, Takasaki K. Age-related decrease of calcitonin gene-related peptide-containing vasodilator innervation in the mesenteric resistance vessel of the spontaneously hypertensive rat. Circ Res 1990; 67: 733-743.

15 Reddy SS, Karuna R, Baskar R, Saralakumari D. Prevention of insulin resistance by ingesting aqueous extract of Ocimum sanctum to fructose-fed rats. Horm Metab Res 2008; 40: 44-49.

16 Han SP, Naes L, Westfall TC. Inhibition of periarterial nerve stimulation-induced vasodilation of the mesenteric arterial bed by CGRP (8-37) and CGRP receptor desensitization. Biochem Biophys Res Commun 1990; 168: 786-791.

17 Kawasaki H, Nuki C, Saito A, Takasaki K. NPY modulates neurotransmission of CGRPcontaining vasodilator nerves in rat mesenteric arteries. Am J Physiol 1991; 261: H683-H690.

18 Nuki C, Kawasaki H, Takasaki K, Wada A. Pharmacological characterization of presynaptic calcitonin gene-related peptide (CGRP) receptors on CGRP-containing vasodilator nerves in rat mesenteric resistance vessels. J Pharmacol Exp Ther 1994; 268: 59-64.

19 Marti E, Gibson SJ, Polak MN, Facer P, Springall DR, Aswegen G, Aitchison M, Koltzenburg M. Ontogeny of peptide- and amine-containing neurons in motor, sensory, 
and autonomic regions of rat and human spinal cord, dorsal root ganglia and rat skin. J Comp Neurol 1987; 266: 332-359.

20 Zamami Y, Takatori S, Goda M, Koyama T, Iwatani Y, Jin X, Takai-Doi S, Kawasaki H. Royal jelly ameliorates insulin resistance in fructose-drinking rats. Biol Pharm Bull 2008; 31: 2103-2107.

21 Kawasaki H, Nuki C, Saito A, Takasaki K. Adrenergic modulation of calcitonin generelated peptide (CGRP)-containing nerve-mediated vasodilation in the rat mesenteric resistance vessel. Brain Res 1990; 506: 287-290.

22 Kawasaki H, Urabe M, Takasaki K. Enhanced 5-hydroxytryptamine release from vascular adrenergic nerves in spontaneously hypertensive rats. Hypertension 1987; 10: 321-327.

23 Kawasaki H, Takasaki K. Vasoconstrictor response induced by 5-hydroxytryptamine released from vascular adrenergic nerves by periarterial nerve stimulation. $J$ Pharmacol Exp Ther 1984; 229: 816-822.

24 Edvinsson L, Emson P, McCulloch J, Tatemoto K, Uddman R. Neuropeptide Y: cerebrovascular innervation and vasomotor effects in the cat. Neurosci Lett 1983; 43: 79-84.

25 Kawasaki H, Nuki C, Saito A, Takasaki K. Role of calcitonin gene-related peptidecontaining nerves in the vascular adrenergic neurotransmission. J Pharmacol Exp Ther 1990; 252: 403-409.
26 Kamiya H, Murakawa Y, Zhang W, Sima AA. Unmyelinated fiber sensory neuropathy differs in type 1 and type 2 diabetes. Diabetes Metab Res Rev 2005; 21: 448-458.

27 Murakawa Y, Zhang W, Pierson CR, Brismar T, Ostenson CG, Efendic S, Sima AA. Impaired glucose tolerance and insulinopenia in the GK-rat causes peripheral neuropathy. Diabetes Metab Res Rev 2002; 18: 473-483.

28 Chi TC, Liu IM, Cheng JT. Less of insulin desensitization in sympathetic nerve terminals from wistar rats with insulin resistance. J Auton Nerv Syst 2000; 80: 80-84.

29 Recio-Pinto E, Rechler MM, Ishii DN. Effects of insulin, insulin-like growth factor-II, and nerve growth factor on neurite formation and survival in cultured sympathetic and sensory neurons. J Neurosci 1986; 6: 1211-1219.

30 Skaper SD, Selak I, Varon S. Molecular requirements for survival of cultured avian and rodent dorsal root ganglionic neurons responding to different trophic factors. $J$ Neurosci Res 1982; 8: 251-261.

31 Zackenfels K, Oppenheim RW, Rohrer H. Evidence for an important role of IGF-I and IGF-II for the early development of chick sympathetic neurons. Neuron 1995; 14: $731-741$. 